\title{
Light confinement in liquid crystals for optofluidic integrated microsystems -INVITED
}

\author{
Antonio d'Alessandro ${ }^{1, *}$ Anju M. Kumaran ${ }^{1}$ \\ ${ }^{1}$ Sapienza University of Rome, Department of Information Engineering, Electronics and Telecommunications, 00184 Rome, Italy
}

\begin{abstract}
In this paper technology to make optical waveguides and microfluidic channels integrated on the same substrate will be reported to envisage novel micro-optofluidic chips. PolyDiMethylSiloxane (PDMS) is used to make microchannels to be filled with biological solutions. Liquid crystals (LC) are used to confine light to produce optical interaction with biological fluidic specimen. Optical waveguides base on PDMS channels filled with LC, named LC:PDMS waveguides, including both straight and bending channels are reported to design photonic devices. Electro-optic effect of LC allows to make tuneable optical waveguides to reconfigure the entire optofluidic microsystem which can include gold nanoparticles for photo-thermal therapies. Coplanar gold electrodes can switch LC molecules with applied voltage of about $2 \mathrm{~V}$. Such electrode configuration can be used to make optical switches and wavelengths demultiplexers. A zero-gap directional coupler based on LC:PDMS waveguides has been designed to switch light from one waveguide to another with an extinction ratio of $16 \mathrm{~dB}$ by applying a voltage of just $1.62 \mathrm{~V}$. A multimode interference demultiplexer has been also designed to demultiplex wavelengths at $980 \mathrm{~nm}$ and $1550 \mathrm{~nm}$ in two output waveguides with an extinction ratio better than $11 \mathrm{~dB}$ by applying about $7 \mathrm{~V}$.
\end{abstract}

\section{Introduction}

Optofluidic channels have been successfully proposed by infiltrating liquid crystals (LC) in silicon grooves [1] and in PolyDiMethylSiloxane (PDMS) [2].

Both the variation in the diffraction pattern of an array of microfluidic channels acting as a switchable grating using ITO electrodes [3] and simple optical waveguides were experimentally demonstrated using LC infiltrated in PDMS, named LC:PDMS waveguides. PDMS channels have been made by using casting and molding technique, which is a low cost process to easily obtain well defined PDMS patterns [4].

PDMS has been demonstrated to be very effective and reliable for soft lithographic fabrication of many microfluidic and micro-optical devices [5]. PDMS with respect to other materials, such as silicon or glass, is the easy and cheap technological lithographic processing needed to make patterned geometries including bends. Flexibility is another interesting feature of PDMS enabling emerging applications which are impossible to achieve using conventional rigid or flexible technologies. Examples can be found in robotics health care, biomedical technologies, and consumer electronics.

Liquid crystals (LC) are effective materials to make both electrically [6] and optically [7-8] controlled optical waveguides to make switchable and reconfigurable waveguides to be used both at the visible and near infrared wavelengths [9]. In particular the efficient electro-optical effect with the additional advantage of negligible electrical current absorption and the nonlinear optical properties allow very low power consumption without significant energy dissipation. Such characteristics make LC-based photonic devices interesting potential candidates for both datacom and optofluidic applications [10]. In this paper we present design of both straight and bending optical waveguides based on LC:PDMS. Moreover some examples of electro-optically controlled devices based on LC:PDMS waveguides combined with coplanar gold electro-plated electrodes are shown. The fabrication process of both waveguides and gold electrodes will be reviewed. A study of LC orientation in PDMS channels based on Monte Carlo simulations can be used. Furthermore the design of an electro-optical switch consisting of a zero gap directional coupler and a multimode interferometer used as a wavelength demultiplexer based on LC:PDMS is presented.

\section{LC: PDMS optical waveguides}

PDMS channels are made by using casting and molding technique. Soft photolithography with SU-8 2005 negative photoresist by Micro Chem is used to create the mold of micro-channels. Waveguides are shaped in a PDMS bulk by pouring the liquid mixture on the SU-8 mold, placed in a holder to host $3 \mathrm{~mm}$ thick PDMS. Then the channels for NLC filling is shaped by pouring the PDMS on a cleaned silicon substrate, in order to provide a very smooth surface for PDMS. The SU8 mold is placed in the PDMS to shape the micro-channels. In a final step, a uniform PDMS film is obtained by spinning the liquid

* Corresponding author: antonio.dalessandro@uniroma1.it 
PDMS on a glass substrate. The PDMS samples, after vacuum treatment to avoid air entrapment in the channels, are baked in oven at $80^{\circ} \mathrm{C}$ for approximately 15 minutes. The sample with micro-channels is then simply peeled from the SU-8 mold and the PDMS in excess will be sharply cut away by using a razor blade. The complete assembled sample, like that shown in Figure 1 as an example, is finally baked at $80^{\circ} \mathrm{C}$ for 60 minutes. The NLC E7 is infiltrated in its isotropic phase at $80^{\circ} \mathrm{C}$ through a hole in a vacuum oven and then cooled down at room temperature. No alignment layer is used to orient the LC molecules. Light transmission through LC:PDMS waveguides has been simulated after calculating the refractive index profile of the $\mathrm{LC}$ related to its orientation. The distribution of the LC orientation inside the PDMS channel has been obtained by minimizing the OseenFrank free energy equation which includes both the elastic and the dielectric components related to an external applied field and solving the Poisson equation by using finite elements through COMSOL Multiphysics ${ }^{\circledR}$, as already performed for other types of LC waveguides [11].

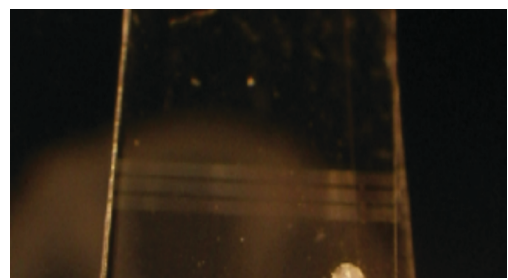

Fig. 1. A set of LC:PDMS waveguides.

The orientation of the LC corresponds to the minimum of the free energy which is given by:

$$
\mathrm{F}=\mathrm{F}_{\mathrm{el}}-\mathrm{F}_{\text {diel }}-\mathrm{F}_{\mathrm{opt}}
$$

which includes the elastic term $\mathrm{F}_{\mathrm{el}}$, the dielectric term $F_{\text {diel }}$ and the optical term $F_{\text {opt }}$ deriving by the electric field of the optical beam itself propagating in the LC.

The elastic energy according to the Oseen-Frank theory is expressed by [34]:

$$
\mathrm{F}_{\mathrm{el}}=\frac{1}{2} \iiint\left[\mathrm{k}_{11}(\nabla \cdot \mathbf{n})^{2}+\mathrm{k}_{22}(\mathbf{n} \cdot \nabla \times \mathbf{n})^{2}+\mathrm{k}_{33}(\mathbf{n} \times \nabla \times \mathbf{n})^{2}\right] \mathrm{dv}
$$

where $\mathrm{k}_{11}, \mathrm{k}_{22}$ and $\mathrm{k}_{33}$ are the elastic constants corresponding to the splay, twist and bend respectively and $\mathrm{dv}$ is the elementary integration volume. The dielectric energy is given by:

$\mathrm{F}_{\mathrm{diel}}=\frac{1}{2} \iiint\left[\epsilon_{0} \epsilon_{\perp, \mathrm{ac}}\left|\mathbf{E}_{\mathrm{ac}}\right|^{2}+\epsilon_{0} \Delta \epsilon_{\mathrm{ac}}\left(\mathbf{E}_{\mathrm{ac}} \cdot \mathbf{n}\right)^{2}\right] \mathrm{dv}$

where $\epsilon_{0}$ is dielectric permittivity in vacuum, $\epsilon_{\perp, a c}$ is the dielectric permittivity perpendicular to the optical axis, $\Delta \epsilon_{\mathrm{ac}}$ is the dielectric anisotropy, and $\mathbf{E}_{\mathrm{ac}}$ is the applied electric field at low frequency. The optical term of the energy is given by:

$\mathrm{F}_{\mathrm{opt}}=\frac{1}{2} \iiint\left[\epsilon_{0} \epsilon_{\perp, \mathrm{opt}}\left|\mathbf{E}_{\mathrm{opt}}\right|^{2}+\epsilon_{0} \Delta \epsilon_{\mathrm{opt}}\left(\mathbf{E}_{\mathrm{opt}} \cdot \mathbf{n}\right)^{2}\right] \mathrm{dv}$ where $\epsilon_{0}$ is dielectric permittivity in vacuum, $\epsilon_{\perp \text {, opt }}$ is the dielectric permittivity perpendicular the optical axis, $\Delta \epsilon_{\mathrm{opt}}$ is the dielectric anisotropy, and $\mathbf{E}_{\text {opt }}$ is the electric field at the optical frequencies. The minimization of $\mathrm{F}$ is achieved by solving the Euler - Lagrange equation. This approach is simpler than global or relaxation approaches, and therefore more adapted for large 3D problems of typical integrated optical structures. To solve the partial derivative equation, a finite element method is well suited since it allows the implementation of the weak form of the Euler - Lagrange equation. The minimization of $\mathrm{F}$ is coupled with the solution of the Poisson equation for the distribution of the electric potential $\Phi$ in the LC structure:

$$
\nabla \cdot\left[\varepsilon_{\perp, \mathrm{ac}} \nabla \Phi+\Delta \epsilon_{\mathrm{ac}}(\nabla \Phi \cdot \mathbf{n}) \mathbf{n}\right]=0
$$

The resulting output is the spatial distribution of the LC director orientation, from which the refractive index profile can be derived. Simulation of light propagation by using RSoft BeamPROP ${ }^{\mathrm{TM}}$ indicates a multimodal behaviour of the waveguide. Figure 2 shows the first order mode for TE light propagating along a straight LC:PDMS waveguide.

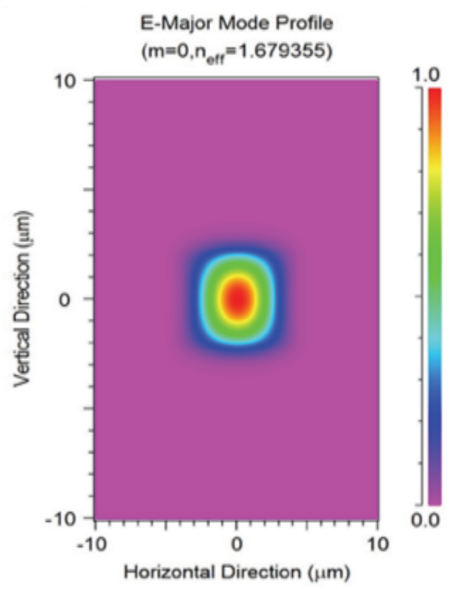

Fig. 2. Contour plots of the first TE-like mode propagating through an LC:PDMS waveguide.

A significant advantage in using LC:PDMS waveguides is that PDMS channels can be patterned to obtain bending paths. Bends are essential to make a large variety of optical circuits including ring resonators with compact dimensions. Figure 3 shows propagation of light though an S-bend with a bend radius of $1.3 \mathrm{~mm}$ over an extension of $10 \mathrm{~mm}$.

Such a bend radius guarantees minimum optical losses as shown in Figure 4 in which optical loss in $\mathrm{dB} / \mathrm{cm}$ and the normalised transmittance versus bend radius are plotted. The power loss is the total sum of the radiation and transmittance losses of the complete S-bend structure obtained from the BPM simulation. For a bend radius of both 1.3 and $1.4 \mathrm{~mm}$ a power loss of $0.622 \mathrm{~dB} / \mathrm{cm}$ has been calculated. Losses increase for bend radii higher than $1.4 \mathrm{~mm}$ but saturating at a value of about $0.628 \mathrm{~dB} / \mathrm{cm}$. Consistently power transmission reaches a peak for a bend radius of 1.3-1.4 $\mathrm{mm}$ and decreases saturating at higher bend radii. 


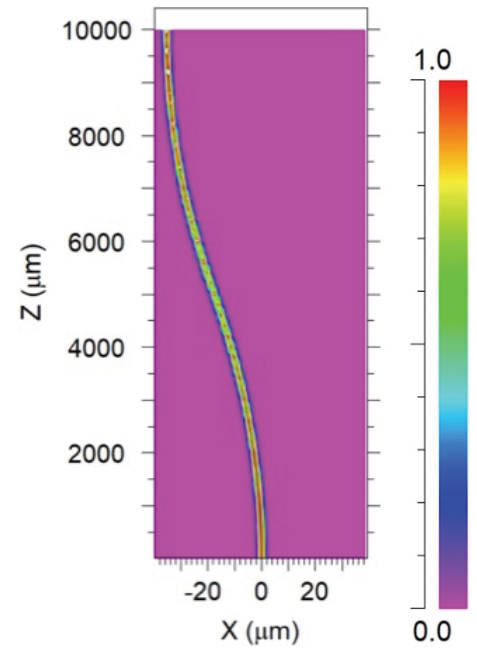

Fig. 3. Optical power propagation through an S-bend LC:PDMS waveguide.

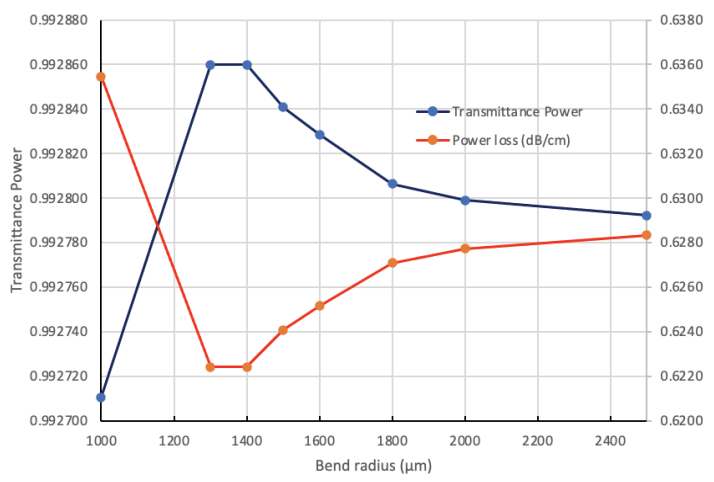

Fig. 4. Normalised optical transmittance and losses versus bend radius in a S-bend LC:PDMS optical waveguide.

Monte Carlo simulation method has been applied to investigate LC molecular orientation [12]. In particular, the Lebwhol and Lasher (LL) [13] model, the prototype potential for the mesoscale simulations of nematic LC, allows to simulate a high number of molecules, represented by three-dimensional headless spins, and then to reproduce or predict observables which can be obtained in real experiments. Some of these experiments are the microscopy polarized images which can give clear indications of the molecular organization inside a nematic LC sample [14].

The optical characterization to check the LC alignment in the LC:PDMS waveguides is performed by using a polarized microscope. Polarized light is transmitted through the channels revealing the homeotropic arrangement of the LC molecules inside the PDMS channels. Such orientation of the LC is proven by the black part in the central region for the waveguides indicating that polarization of input light is not changed when transmitted through the LC and is stopped by the crossed analyser. Light leaks through the edges of the waveguides because of the optical retardation induced by the LC molecules homeotropically aligned on the vertical sides of the PDMS channels.

The homeotropic alignment of the LC inside the PDMS channel creates a distribution of the LC refractive index which allows polarization independent propagation of light at both visible and near infrared wavelengths. Standard butt coupling technique is used to inject light beam into the LC:PDMS waveguides by using cleaved single-mode optical fibers. Both visible and near infrared light sources were used.

Figure 5 shows that it is possible to couple and transmit visible light. Near infrared wavelengths have been also injected and polarization independence of light propagation has been observed as expected due to the homeotropic alignment of LC molecules.

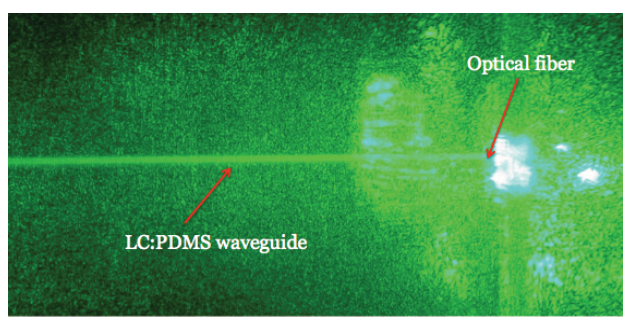

Fig. 5. Green light propagation in an LC:PDMS waveguide.

It is also possible to reorient LC molecules by applying an electric field between coplanar electrodes, as shown in Figure 6 . The electrodes can be deposited by means of electroplating. It has been shown that a voltage as low as $1.8 \mathrm{~V}$ is enough to switch the LC molecules between the coplanar electrodes.

Such electrode configuration can be proposed to make electro-optical switchable LC:PDMS waveguide based devices.

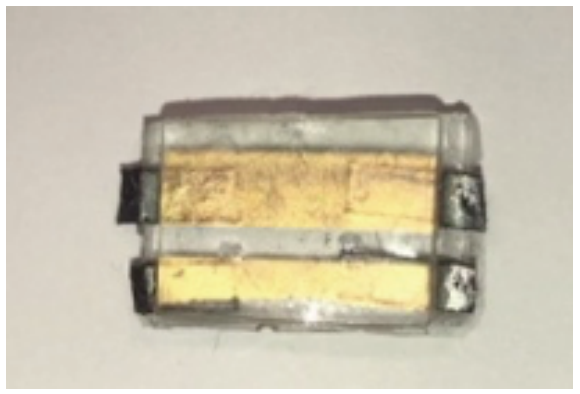

Fig. 6. Gold plated coplanar electrodes embedded in a two layers of PDMS [14].

\section{Device applications and future developments}

Photonic devices based on LC:PDMS waveguides have been demonstrated such as directional couplers, optical switches, multimode interferometers (MMI). In an electro-optical controlled directional coupler with zero gap, as shown in left-hand side of Figure 7, it is possible to switch light from one waveguide to a second one with 
an extinction ratio of $16 \mathrm{~dB}$ with an applied voltage of just $1.62 \mathrm{~V}$. Light remains in the same waveguide with an extinction ratio of about $18 \mathrm{~dB}$ with a voltage of $1.76 \mathrm{~V}$. A MMI based on LC:PDMS technology, as sketched in the right-hand side of Figure 7, has been also designed to demultiplex wavelengths at $980 \mathrm{~nm}$ and $1550 \mathrm{~nm}$ in two output waveguides with an extinction ratio better than 11 $\mathrm{dB}$ by applying about $7 \mathrm{~V}$.

LC:PDMS based devices can be also used for biochip applications. One example consists of biochip for bacterial killing through the temperature increase caused by the heating of gold nanoparticles. Such a biochip can be made of PDMS in which microfluidic channels, gold nanorods (GNRs) and optical waveguides are combined and integrated.

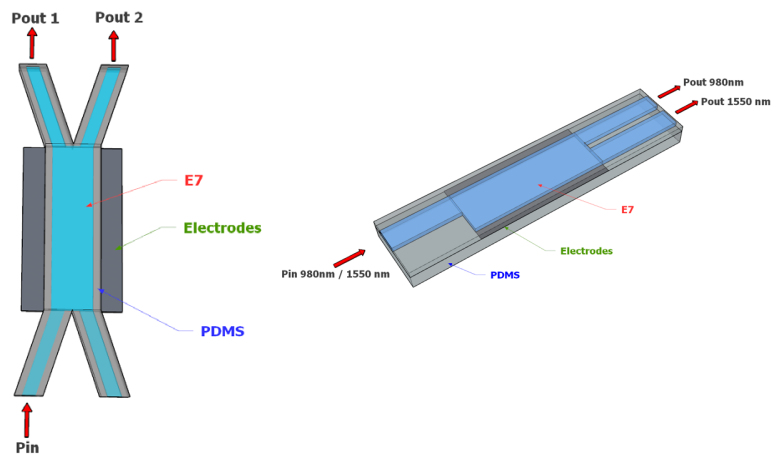

Fig. 7. (Left) Zero-gap directional coupler based and (right) tuneable MMI based on LC:PDMS waveguides [14].

\section{References}

1. D. Donisi, B. Bellini, B., R. Beccherelli, R. Asquini, G. Gilardi, M. Trotta, A. d'Alessandro, IEEE J. Quantum Electron. 46, 762 (2010)

2. A. E. Vasdekis, J. Cuennet, D. Psaltis, Proc. SPIE 8475, 847507 (2012)

3. L. De Sio, M. Romito, M. Giocondo, A. E. Vasdekis, A. De Luca, Lab on a chip 12, 3760 (2012)

4. A. d'Alessandro, L. Martini, G. Gilardi, R. Beccherelli and R. Asquini, IEEE Photon. Technol. Lett. 27, 1709 (2015)

5. D. A. Chang-Yen, R. K. Eich, B. K. Gale, J. Light. Technol. 23, 2088 (2005)

6. D. Donisi, B. Bellini, R. Beccherelli, R. Asquini, G. Gilardi, M. Trotta, A. d'Alessandro, IEEE J. Quantum Electron. 46, 762 (2010)

7. A. d'Alessandro, R. Asquini, M. Trotta, G. Gilardi, R. Beccherelli, I. C. Khoo, Appl. Phys. Lett. 97, 093302 (2010)

8. J.F. Henninot, M. Debailleul, R. Asquini, A. d'Alessandro, M. Warenghem, J. Opt. A: Pure Appl. Opt. 6, 315 (2004).

9. I. C. Khoo, Liquid Crystals, (Wiley, 2nd Ed., 2005)
10. D. C. Zografopoulos, R. Asquini, E. Kriezis, A. d'Alessandro, R. Beccherelli, Lab on a chip 12, 3598 (2012)

11. A. d'Alessandro, L. Martini, L. Civita, R. Beccherelli, R. Asquini, Proc. SPIE 9384, 93840L (2015)

12. P. Pasini, C. Chiccoli, C. Zannoni, Advances in the computer simulations of liquid crystals, (Kluwer, 2000)

13. P. A. Lebwohl, G. Lasher, Phys. Rev. A. 6, 426 (1972)

14. R. Asquini, C. Chiccoli, P. Pasini, L. Civita, A. d'Alessandro, Liq. Cryst. 45, 1 (2018) 